\title{
Tradução e adaptação transcultural do Groningen Frailty Indicator para idosos brasileiros
}

\section{Groningen Frailty Indicator transcultural translation and adaptation for elderly Brazilians}

\author{
Cíntia Lira Borges ${ }^{1}\left(\mathbb{D}\right.$, Bruna Karen Cavalcante Fernandes ${ }^{2}\left(\mathbb{D}\right.$, Jorge Wilker Bezerra Clares $^{3} \mathbb{C}$, Maria Lígia Silva Nunes \\ Cavalcante $^{2}$ (D) , Saul Filipe Pedrosa Leite ${ }^{4}$ (D) , Arnaldo Aires Peixoto Junior ${ }^{5,6}$ (D) , Maria Célia de Freitas ${ }^{7}$ (D)
}

1. Doutora em Saúde Coletiva da Universidade Estadual do Ceará (UECE), Fortaleza, CE, Brasil. 2. Doutoranda em Cuidados Clínicos em Enfermagem e Saúde da Universidade Estadual do Ceará (UECE), Fortaleza, CE, Brasil. 3. Mestre em Cuidados Clínicos e Enfermagem e Saúde da Universidade Estadual do Ceará (UECE), Fortaleza, CE, Brasil. 4. Médico da Estratégia de Saúde da Família da Prefeitura de Fortaleza, Fortaleza, CE, Brasil. 5. Docente da Faculdade de Medicina da Universidade Federal do Ceará (UFC), Fortaleza, CE, Brasil. 6. Docente do curso de Medicina do Centro Universitário Christus (UNICHRISTUS), Fortaleza, CE, Brasil. 7. Docente da Universidade Estadual do Ceará (UECE), Fortaleza, CE, Brasil.

\section{Resumo}

Objetivo: realizar a tradução, a adaptação transcultural e a validação de conteúdo do Groningen Frailty Indicator para aplicação em idosos brasileiros. Método: os procedimentos seguiram de tradução, obtenção da versão consensual da tradução, retrotradução, validação de conteúdo e pré-teste. A versão final em português foi aplicada a 30 idosos. Resultados: a versão brasileira do Groningen Frailty Indicator conservou a equivalência semântica. A população-alvo avaliou todos os itens como de fácil compreensão. Entretanto, faz-se necessário avaliar a equivalência de mensuração, validade externa e reprodutibilidade. Conclusão: os resultados sugerem que o Groningen Frailty Indicator traduzido e adaptado para a cultura brasileira é um instrumento de fácil aplicação, tornando-o viável para a prática clínica com idosos.

Palavras-chave: Idoso Fragilizado. Envelhecimento. Estudos de Validação. Comparação Transcultural. Inquéritos e Questionários.

\begin{abstract}
Objective: to perform translation, cross-cultural adaptation and content validation of the Groningen Frailty Indicator for application in Brazilian elderly Method: The procedures followed from translation, obtaining the consensual version of the translation, back translation, content validation and pre-test. The final version in Portuguese was applied to 30 elderly people. Results: The Brazilian version of the Groningen Frailty Indicator preserved the semantic equivalence. The target population evaluated all items as being easily understood. However, it is necessary to evaluate its equivalence of measurement, external validity and reproducibility. Conclusion: The results suggest that the Groningen Frailty Indicator translated and adapted to the Brazilian culture is an instrument of easy application, making it feasible for clinical practice with the elderly.
\end{abstract}

Key words: Frail elderly. Aging. Validation studies. Cross-cultural comparison. Surveys and questionnaires

\section{INTRODUÇÃO}

O crescimento da população idosa no Brasil tem representado um desafio às políticas públicas, sobretudo no setor saúde, uma vez que o envelhecimento populacional vem acompanhado por uma maior incidência das doenças crônicas, as quais contribuem para a dependência física e social entre os idosos ${ }^{1}$.

Diversas condições se associam à saúde dos idosos, entre as quais se destaca a síndrome de fragilidade. Esta é considerada uma síndrome clínica muito prevalente, que aumenta com a idade e acarreta maior vulnerabilidade aos fatores estressantes que resultam no declínio das reservas fisiológicas e das habilidades para executar as atividades de vida diária2. Ressalta-se que os idosos frágeis apresentam maior vulnerabilidade para quedas, incapacidade, hospitalizações e morte ${ }^{3-4}$.

Diante de sua importância clínica, torna-se necessário realizar a detecção precoce da fragilidade em pessoas idosas, a fim de orientar a tomada de decisões e o planejamento de ações assistivas e de educação, prevenção e promoção da saúde. Para isso, é fundamental a existência de instrumentos que ajudem a identificar os indicadores de fragilidade em idosos, possibilitando 0 desenvolvimento de intervenções mais adequadas e específicas a essa população $0^{5}$.

Entre os instrumentos para avaliação da fragilidade propostos, optou-se, no presente estudo, pela tradução e adaptação transcultural do Groningen Frailty Indicator (GFI) para o português do Brasil, por ser um instrumento prático e de fácil manuseio para determinar o nível de fragilidade. Foi desenvolvido na Holanda por Steverink e colaboradores, sendo composto por 15 itens divididos em quatro domínios do funcionamento: físicos (nove itens), cognitiva (um item), social

Correspondente: Cíntia Lira Borges. Av: Filomeno Gomes, número 860, ap: 703, bairro: Jacarecanga CEP: 60010-281. Fortaleza (CE), Brasil. E-mail: cintialiraborges@yahoo.com.br

Conflito de interesse: Não há conflito de interesse por parte de qualquer um dos autores.

Recebido em: 13 Jul 2018; Revisado em: 8 Abr 2019; 29 Abr 2019; Aceito em: 2 Mai 2019 
(três itens) e psicológicos (dois itens). A maioria dos itens pode ser respondida com "sim" ou "não"; sendo também possível responder com a opção "às vezes" para os itens relativos aos domínios cognitivo e psicossocial. A pontuação varia de 0 a 15, sendo de 0 a 3 idoso não frágil, e igual ou superior a 4 idoso frágil.

Sua aplicação poderá ser útil ao rastreamento da fragilidade em idosos, subsidiando o planejamento de estratégias terapêuticas adequadas para a prevenção de desfechos clínicos negativos. Nessa perspectiva, objetivou-se realizar a tradução, adaptação transcultural e validação de conteúdo do GFI para aplicação em idosos brasileiros.

\section{MÉTODOS}

Trata-se de uma pesquisa metodológica que discorre sobre o processo de tradução e adaptação transcultural do GFI. Destacase que o estudo do tipo metodológico envolve investigação dos métodos de obtenção, organização de dados e conclusão de pesquisa rigorosa de desenvolvimento, validação e avaliação de ferramentas e métodos de pesquisa ${ }^{7}$.

Para que uma escala seja adaptada culturalmente para outro país, é necessário seguir uma metodologia rigorosa e específica. $O$ estudo em questão utilizou o método de adaptação transcultural proposto por Beaton et al. $(2007)^{8}$ que teve apoio da Academia Americana de Cirurgiões Ortopédicos (AAOS) na criação das diretrizes: "Recomendações para Adaptação Cultural de Medidas de Estados de Saúde". Este modelo segue os pressupostos de Guillemin et al. (1993) ${ }^{9}$ que recomenda que os itens de um instrumento de diferente idioma não devem ser somente traduzidos linguisticamente, mas adaptados culturalmente de acordo com a realidade do outro lugar. 0 processo transcultural é uma tarefa complexa e demorada, mas extremamente importante para o avanço da compreensão do alcance e dos limites das construções teóricas e dos quadros em diferentes realidades culturais ${ }^{10}$.

Ressalta-se que o estudo foi realizado após a obtenção do consentimento formal da autora do instrumento original e seguiu os procedimentos indicados ${ }^{8}$ : tradução, obtenção da versão consensual da tradução, retrotradução, validação de conteúdo e pré-teste.

Inicialmente, o instrumento original foi traduzido por dois profissionais brasileiros com fluência no idioma holandês, de forma independente e simultânea. Somente um dos tradutores foi informado quanto aos objetivos do instrumento e os conceitos implicados, de modo a favorecer a equivalência cultural e idiomática. $O$ segundo profissional não recebeu essas informações para a extração de significados inesperados do instrumento original.

A etapa seguinte foi realizada por um dos autores (CLB) e por um terceiro tradutor brasileiro com domínio na língua holandesa, com o objetivo de unificar as versões de tradução e obter consenso referente ao conteúdo explorado.

A versão consensual em português foi traduzida para a língua original (retrotradução) por dois tradutores de nacionalidade holandesa com fluência na língua portuguesa, que trabalharam de forma simultânea e independente, e não participaram da primeira etapa. Os tradutores não foram informados sobre os objetivos da tradução, não conheciam o instrumento original nem possuíam formação acadêmica na área.

Na etapa seguinte, um comitê multidisciplinar composto por dois profissionais (uma enfermeira e um filósofo) com vasta experiência na área de metodologia científica; uma enfermeira, com experiência assistencial e de docência na área de saúde do idoso; a pesquisadora e sua orientadora; e o profissional que participou da etapa de consolidação da versão final da tradução realizou a revisão e comparação entre as traduções finais obtidas.

A equipe de profissionais formada nessa etapa entrou em concordância com relação a todos os itens, consolidando todas as versões em uma versão pré-final para testes de campo. Nesse estágio, os juízes avaliaram equivalências entre a fonte e a versão de destino, atentando para ${ }^{9}$ equivalência semântica (sentido das palavras); equivalência idiomática (expressão popular); equivalência experimental (vivência/experiência do cotidiano); equivalência conceitual (pertinência dos conceitos e dimensões assimilados na cultura da versão traduzida).

A etapa de adaptação transcultural foi composta por análise de conteúdo da escala por um comitê de especialistas e um pré-teste envolvendo 30 idosos. O comitê de especialistas foi composto por 19 enfermeiros, um médico, um fisioterapeuta e um nutricionista, com experiência assistencial ou com docência em saúde do idoso.

A avaliação dos itens pelos juízes foi realizada mediante uma escala do tipo Likert ( 1 = não representativo, 2 = necessita de grande revisão para ser representativo, $3=$ necessita de pequena revisão para ser representativo, $4=$ representativo). Além disso, caso julgassem necessário, poderiam sugerir modificações e correções, explicitando a razão, com a intenção de melhorar a adaptação ao contexto brasileiro, visando manter uma linguagem simples, melhorar a compreensão dos itens e aumentar sua clareza. Dessa forma, foi cumprida a etapa de equivalência semântica, idiomática, experimental e conceitual. Utilizou-se o índice de validade de conteúdo (IVC) para avaliar a concordância entre os juízes.

Um pré-teste foi realizado com uma amostra de conveniência de 30 idosos, no período de agosto a setembro de 2013, com o objetivo de identificar possíveis dificuldades de compreensão derivadas da forma de aplicação, da forma de apresentação, do conteúdo das questões e das alternativas de respostas. Os dados foram coletados por entrevistadores treinados pela pesquisadora para o protocolo desta pesquisa. Ao término da aplicação, foi solicitado ao idoso que referisse suas sugestões, 
caso percebesse alguma dificuldade para a compreensão dos itens que compõem o instrumento. A repetição de itens pelos entrevistados demonstrou confiabilidade das respostas ${ }^{11}$. Essa etapa foi importante para assegurar que a versão adaptada retém sua equivalência no momento da aplicação.

O projeto foi aprovado pelo Comitê de Ética em Pesquisa da Universidade Estadual do Ceará, mediante parecer no. 305.456 e CAAE 12390513.8.0000.5534. Todos os participantes assinaram o Termo de Consentimento Livre e Esclarecido.

\section{RESULTADOS}

O processo de tradução e adaptação transcultural desse instrumento seguiu a metodologia proposta na literatura ${ }^{8}$. As alterações dos itens foram realizadas baseadas nas sugestões do comitê de especialistas, com o objetivo de melhorar a clareza e a interpretação para população-alvo. As etapas da tradução e validação de conteúdo estão apresentadas no quadro 1.

Quadro 1. Avaliação da equivalência semântica entre a versão original, a versão-síntese das traduções e a versão final adaptada para o português (Brasil) do Groningen Frailty Indicator. Fortaleza-CE, 2013.

\begin{tabular}{|c|c|}
\hline Versão original & Versão-síntese das traduções \\
\hline $\begin{array}{l}\text { 1. Kunt u geheel zelfstandig buitenshuis } \\
\text { rondlopen (rondom huis of naar de } \\
\text { buren)? }\end{array}$ & $\begin{array}{l}\text { 1. O(a) Sr. (a) consegue fazer suas compras } \\
\text { de forma independente? }\end{array}$ \\
\hline $\begin{array}{l}\text { 2. Kunt u geheel zelfstandig buitenshuis } \\
\text { rondlopen (rondom huis of naar de } \\
\text { buren)? }\end{array}$ & $\begin{array}{l}\text { 2. O(a) Sr.(a) consegue caminhar fora de } \\
\text { casa de forma independente (ao redor da } \\
\text { sua casa ou ir até seus vizinhos)? }\end{array}$ \\
\hline
\end{tabular}

3. Kunt u zich geheel zelfstandig aan- en uitkleden?

4. Kunt u geheel zelfstandig van en naar het toilet gaan?

Met zelfstandig bedoelen we: zonder enige vorm van hulp van iemand. Gebruik maken van hulpmiddelen als stok, rollator, rolstoel, geldt als zelfstandig.

5. Als $u$ een rapportcijfer zou moeten geven voor uw lichamelijke fitheid, waarbij een 1 staat voor heel slecht, en een 10 staat voor uitstekend, wat zou dit cijfer dan zijn?
3. O(a) Sr.(a) consegue se vestir e despir de forma independente?
Versão final em português

1. O(a) Sr.(a) consegue fazer suas compras (exemplo: em supermercados, farmácias, mercadinhos, shoppings) sozinho(a), sem a ajuda de outras pessoas?

2. O(a) Sr.(a) consegue caminhar fora de casa, nas proximidades onde mora (ao redor de sua casa ou na casa de seus vizinhos), sozinho(a), sem a ajuda de outras pessoas?

3. O(a) Sr.(a) consegue se vestir e tirar suas roupas sozinho(a), sem a ajuda de outras pessoas?

4. O(a) Sr.(a) consegue ir e sair do banheiro de forma independente?

É considerada, de forma independente, não precisar de ajuda de outra pessoa ou fazer uso de utensílios de apoio como bengala, andador e cadeira de rodas.

5. Se o(a) Sr.(a) tivesse que dar uma nota para a sua capacidade física, sendo a nota número 1 para muito ruim e a nota número 10 para excelente, qual seria este número?

4. O(a) Sr.(a) consegue entrar no banheiro e sair dele sozinho(a), sem a ajuda de outras pessoas, mesmo fazendo uso de andador, bengala, muleta ou cadeira de rodas?

5. Se o(a) Sr.(a) tivesse que dar uma nota para a sua condição física de 0 a 10 , sendo a nota no 0 para muito ruim e a nota $n=10$ para excelente, qual seria este número?
Nota: Considerar condição física como sinônimo de capacidade física e capacidade funcional. Esses termos avaliam a capacidade e/ou aptidão que o idoso tem para realizar determinada ação, como subir obstáculos, praticar atividade física, realizar as atividades diárias no ambiente em que mora (lavar louça, varrer, lavar roupa, limpar a casa, cozinhar, entre outros).

6. O (a) Sr. (a) possui dificuldades para realizar suas atividades diárias por não enxergar bem? diária, devido ao fato de o Sr. (a) enxergar mal?

7. O(a) Sr.(a) encontra problemas na vida diária, devido ao fato de o Sr. (a) ouvir mal?
7. O(a) Sr.(a) possui dificuldades para realizar suas atividades diárias por não ouvir bem? 


\begin{tabular}{|c|c|c|}
\hline Versão original & Versão-síntese das traduções & Versão final em português \\
\hline $\begin{array}{l}\text { 8. Bent } u \text { de afgelopen } 6 \text { maanden veel } \\
\text { afgevallen zonder dat } u \text { dat wilde? }\end{array}$ & $\begin{array}{l}\text { 8. O(a) Sr.(a) emagreceu muito nos } \\
\text { últimos } 6 \text { meses sem que o Sr. (a) } \\
\text { quisesse? }\end{array}$ & $\begin{array}{l}\text { 8. O(a) Sr.(a) emagreceu muito nos } \\
\text { últimos } 6 \text { meses sem que o senhor(a) } \\
\text { quisesse? } \\
\text { Nota: Observar que a perda de mais de } 5 \\
\text { kg no último ano é considerada um dos } \\
\text { sinais clínicos da síndrome da fragilidade. }\end{array}$ \\
\hline $\begin{array}{l}\text { 9. Gebruikt u op dit moment } 4 \text { of meer } \\
\text { verschillende soorten medicijnen? }\end{array}$ & $\begin{array}{l}\text { 9. O(a) } \mathrm{Sr} \text { (a) atualmente toma } 4 \text { ou mais } \\
\text { tipos diferentes de medicamentos? }\end{array}$ & $\begin{array}{l}\text { 9. } \mathrm{O}(\mathrm{a}) \mathrm{Sr} .(\mathrm{a}) \text {, atualmente, toma } 4 \text { ou mais } \\
\text { tipos diferentes de medicamentos? }\end{array}$ \\
\hline 10. Heeft u klachten over uw geheugen? & $\begin{array}{l}\text { 10. O(a) Sr.(a) tem queixas sobre a sua } \\
\text { memória? }\end{array}$ & $\begin{array}{l}\text { 10. O(a) Sr.(a) tem queixas sobre sua } \\
\text { memória (esquece fácil ou possui } \\
\text { dificuldade de memorização)? }\end{array}$ \\
\hline $\begin{array}{l}\text { 11. Ervaart u wel eens een leegte om u } \\
\text { heen? }\end{array}$ & $\begin{array}{l}\text { 11. O(a) Sr.(a) costuma sentir um vazio } \\
\text { em torno do Sr.(a)? }\end{array}$ & $\begin{array}{l}\text { 11. O(a) Sr.(a) costuma sentir um vazio } \\
\text { em torno de sua vida? } \\
\text { Nota: O sentido de vazio pode ser } \\
\text { interpretado como sentir falta de algo } \\
\text { que não sabe o que é, ou sentir angústia, } \\
\text { ou sentir uma falta de sentido em sua } \\
\text { vida. }\end{array}$ \\
\hline 12. Mist u wel eens mensen om u heen? & $\begin{array}{l}\text { 12. O(a) Sr.(a) costuma sentir falta de } \\
\text { pessoas ao seu redor? }\end{array}$ & $\begin{array}{l}\text { 12. O(a) Sr.(a) costuma sentir falta de } \\
\text { pessoas ao seu redor? }\end{array}$ \\
\hline $\begin{array}{l}\text { 13. Voelt } u \text { zich wel eens in de steek } \\
\text { gelaten? }\end{array}$ & $\begin{array}{l}\text { 13. O(a) Sr.(a) costuma se sentir } \\
\text { abandonado? }\end{array}$ & $\begin{array}{l}\text { 13. O(a) Sr.(a) costuma se sentir } \\
\text { abandonado (a)? }\end{array}$ \\
\hline $\begin{array}{l}\text { 14. Heeft u zich de laatste tijd somber of } \\
\text { neerslachtig gevoeld? }\end{array}$ & $\begin{array}{l}\text { 14. O(a) Sr.(a), ultimamente, tem se } \\
\text { sentido triste ou deprimido(a)? }\end{array}$ & $\begin{array}{l}\text { 14. O(a) Sr.(a), ultimamente, tem se } \\
\text { sentido triste ou deprimido (a)? }\end{array}$ \\
\hline $\begin{array}{l}\text { 15. Heeft u zich de laatste tijd nerveus of } \\
\text { angstig gevoeld? }\end{array}$ & $\begin{array}{l}\text { 15. O(a) Sr.(a), ultimamente, tem se } \\
\text { sentido nervoso(a) ou ansioso(a)? }\end{array}$ & $\begin{array}{l}\text { 15. O(a) Sr.(a), ultimamente, tem se } \\
\text { sentido nervoso(a) ou ansioso(a)? }\end{array}$ \\
\hline
\end{tabular}

Observa-se, nos itens 1 a 4, que o termo "independente" foi substituído pela expressão "sozinho(a), sem a ajuda de outras pessoas", a fim de facilitar a compreensão das perguntas, considerando o nível socioeconômico da população alvo da utilização da escala. No item 1 foram acrescentados exemplos de locais para a realização de compras. Foi modificado "ao redor da sua casa ou ir até seus vizinhos" por "ao redor de sua casa ou na casa de seus vizinhos" (item 2); "despir" por "tirar" (item 3); e "ir" por "entrar" (item 4).

No item 4, a nota explicativa sobre a definição de "de forma independente" para pessoas que necessitam de auxíliolocomoção foi integrada à redação do item na versão final do instrumento traduzido. No item 5 , foi modificada a "capacidade física" por "condição física" e acrescentada uma nota com a definição deste termo; também foi modificada a pontuação do item para de "0 a 10 ".

A redação final dos itens 6 e 7 foi alterada, pois a tradução literal poderia dificultar a compreensão da pergunta pelos idosos. No item 8, foi acrescentada uma nota explicativa. No item 10 , houve necessidade de se introduzir a expressão "esquece fácil ou possui dificuldade de memorização". 0 item 11 exigiu certa atenção, uma vez que o termo "vazio" pode apresentar várias interpretações. Portanto, optou-se por inserir uma nota explicativa, a fim de facilitar a compreensão do item (quadro 1).
$\mathrm{Na}$ análise dos escores obtidos dos avaliadores sobre o instrumento todos os itens obtiveram IVC $\geq 0,80$, que corresponde à validade satisfatória e aceitável, apontando que a escala está adequada para aplicação individual em idosos brasileiros.

No pré-teste, nenhum dos participantes necessitou de auxílio para responder aos itens do instrumento, o que foi confirmado por meio do relato dos idosos, que não apontaram dificuldades de entendimento.

\section{DISCUSSÃO}

Nas últimas décadas, pesquisadores desenvolveram medidas de avaliação da fragilidade com o objetivo de ampliar a compreensão e definir operacionalmente seu conceito. No Brasil, há ampla utilização do fenótipo de fragilidade desenvolvido pelo Cardiovascular Health Study ${ }^{12}$. Outros instrumentos utilizados são a Edmonton Frail Scale13, o Tilburg Frailty Indicator14 e o Kihon Checklist15, que foram adaptados e validados para a cultura brasileira e considerados confiáveis e viáveis para uso em idosos. Ressalta-se que é fundamental a existência de instrumentos adaptados e validados que auxiliem os profissionais de saúde na detecção precoce da fragilidade em idosos, possibilitando o delineamento de intervenções efetivas, o que resultará na otimização da qualidade da assistência. 
Nesse contexto, realizou-se a adaptação transcultural do GFI para português do Brasil, mediante rigorosos padrões metodológicos, seguindo as recomendações da literatura especializada8. Isso possibilitou adaptar à realidade dos idosos brasileiros uma escala construída e validada na língua holandesa, que aborda questões específicas para uma avaliação rápida e prática do diagnóstico clínico da síndrome de fragilidade.

A versão traduzida do GFI manteve o nome original em inglês acrescido de "versão brasileira", e sua adequação para o português do Brasil foi avaliada, tanto sob a perspectiva dos especialistas da área da saúde (validação de conteúdo), como sob a perspectiva dos idosos (validação de face), buscando a maior representatividade possível das atividades para a população de interesse.

Destaca-se que a validação de conteúdo avalia em que medida o instrumento possui itens apropriados para medir o constructo específico e cobrir seu domínio7. Já a validade de face é indicada para identificar se o instrumento está verificando o constructo na visão daqueles para os quais serão aplicadas as escalas7. Esse processo tem a finalidade de manter a versão adaptada por profissionais equivalente a uma situação real na qual será subsumida. O questionário deve ser entendido por um adolescente de até 12 anos8, por isso deve ser simples e de fácil compreensão.

Durante todo o processo de equivalência semântica, idiomática e conceitual, foi enfatizada a influência do nível sociocultural e da escolaridade na compreensão das questões do instrumento pelo público-alvo. Assim, foram realizadas modificações na redação de alguns itens da versão traduzida para simplificá- la gramaticalmente e, em alguns casos, para adequá-la a uma linguagem mais coloquial, a fim de melhorar a clareza e facilitar sua compreensão, buscando aproximar o instrumento ao máximo da realidade dos idosos brasileiros.

De acordo com a literatura8, a fase de pré-teste assegura manter a versão original, melhorar a compreensão, encontrar erros e problemas na aplicação do instrumento. No presente estudo, a versão adaptada do GFI apresentou elevado percentual de entendimento e teve boa aceitação dos itens do instrumento, o que foi comprovado pelo relato verbal dos idosos que participaram do pré-teste.

Os limites do estudo foram a dificuldade durante o processo de tradução, no que tange à escala, objeto desta pesquisa, foi traduzida do holandês, e, no Brasil, o idioma não é explorado, comparado ao inglês e ao espanhol, e poucas são as pessoas que possuem domínio da língua; bem como não possibilitou a avaliação das propriedades psicométricas do instrumento.

\section{CONCLUSÃO}

Com este estudo, cumpriu-se uma etapa crucial para disponibilizar um instrumento adaptado para mensurar o nível de fragilidade em idosos. Os resultados sugerem que o GFI traduzido e adaptado para a cultura brasileira é um instrumento de fácil aplicação, tornando-o viável para a prática clínica com idosos. Entretanto, é necessário que essa versão continue a ser testada quanto à avaliação das propriedades psicométricas, estudo de análise fatorial da escala, bem como a análise da consistência interna e a validade de constructo em diferentes contextos socioculturais da realidade brasileira.

\section{REFERÊNCIAS}

1. Miranda GMD, Mendes ACG, Silva ALA. O envelhecimento populacional brasileiro: desafios e consequências sociais atuais e futuras. Rev. bras. geriatr. gerontol [internet]. 2016 Jun [acesso 2019 Abr 25]; 19(3):507-519. Disponível em: http://www.scielo.br/scielo.php?script=sci_arttext\&pid=S180998232016000300507\&lng=pt doi: http://dx.doi.org/10.1590/180998232016019.150140 .

2. Gibson JA, Crowe S. Frailty in Critical Care: Examining Implications for Clinical Practices. Crit Care Nurse [internet]. 2018 [acesso 2019 abril de 25]; 38(3):2935. Disponível em: http://ccn.aacnjournals.org/content/38/3/29.abstract doi: http://dx.doi.org/10.4037/ccn2018336

3. Wen YC, Chen LK, Hsiao FY. Predicting mortality and hospitalization of older adults by the multimorbidity frailty index. PLoS One [internet]. 2017 Nov [acesso 2019 Abr 25] 16;12(11):e0187825. Disponível em: https://www. ncbi.nlm.nih.gov/pmc/articles/PMC5690585/ doi: http://dx.doi.org/10.1371/ journal.pone.0187825

4. Chang SF, Lin PL. Frail phenotype and mortality prediction: a systematic review and meta-analysis of prospective cohort studies. Int J Nurs Stud. [internet]. 2015 Aug [acesso 2019 Abr 25]; 52(8):1362-74. Disponível em: https://www-sciencedirect.ez76.periodicos.capes.gov.br/science/article/ pii/S0020748915001066?via\%3Dihub. doi: http://dx.doi.org/10.1016/j. ijnurstu.2015.04.005.

5. Fabrício-Wehbe SCC, Cruz IR, Haas VJ, Diniz MA, Dantas RAS, Rodrigues RAP. Reproducibility of the Brazilian version of the Edmonton Frail Scale for elderly living in the community. Rev Latino-Am Enfermagem. [Internet]. $2013 \mathrm{Nov-Dez}$ [acesso 2018 Jul 10]; 21(6):1330-6. Disponível em: http://www.scielo.br/scielo. php?script=sci_arttext\&pid=S0104-11692013000601330\&Ing=en doi: http:// dx.doi.org/10.1590/0104-1169.2933.2371

6. Steverink N, Slaets JPJ, Schuurmans H, Van Lis M. Measuring frailty: development and testing of the Groningen Frailty Indicator (GFI). Gerontologist [internet]. 2001 Jan [acesso 2018 Jul 10]; 41: 236-237. Disponível em: https:// www.rug.nl/research/portal/en/publications/measuring-frailty(f91ecfcc-18e0481f-84a7-1230d62e032c).html.

7. Polit DF, Beck CT. Fundamentos de pesquisa em enfermagem: avaliação de evidências para a prática da enfermagem. 7 ed., Porto Alegre: Artmed; 2011.

8. Beaton DE, Bombardier C, Guillemin F, Ferraz MB. Guidelines for the process of cross-cultural adaptation of self-report measures. Spine (Phila Pa 1976). [internet]. 2000 [acesso 2018 Jul 10]; 25(24): 3186-91. Disponível em: https:// journals.Iww.com/spinejournal/Citation/2000/12150/Guidelines_for_the_ Process_of_Cross_Cultural.14.aspx.

9. Guillemin F, Bombardier C, Beaton D. Cross-cultural adaptation of health related quality of life measures: literature review and proposed guidelines. J Clin Epidemiol [Internet]. 1993 Dec [acesso 2019 abril de 25]; 46(12): 14171432. Disponível em: https://www.ncbi.nlm.nih.gov/pubmed/8263569. doi: https://doi.org/10.1016/0895-4356(93)90142-N

10. Craig C, Douglas S. Assessing Cross-Cultural Marketing Theory and Research: 
A Commentary Essay. Journal of Business Research [internet]. 2011 Jun [2019 abril 25];64(6):625-627. Disponível em: https://ideas.repec.org/a/eee/jbrese/ v64y2011i6p625-627.html.

11. Constant HMRM, Figueiró LR, Signor LB, Bisch NK, Barros HMT, Ferigolo M. Tradução, adaptação transcultural e validação de conteúdo da versão em português do Coping Behaviours Inventory (CBI) para a população brasileira. Cad Saúde Pública. [Internet] 2014 [acesso em 29 de Mar 2019]; 30(10): 2049-56. Disponível em: http://www.scielo.br/scielo.php?script=sci_arttext\&pid=S0102311X2014001002049\&lng=en doi: http://dx.doi.org/10.1590/0102$311 \times 00176513$.

12. Fried LP, Tangen CM, Walston J, Newman AB, Hirsch C, Gottdiener J, et al. Frailty in older adults: evidence for a phenotype. J Gerontol A Biol Sci Med Sci. [Internet]. 2001 Mar [acesso 2018 Jul 10]; 56(3): 146-54. Disponível em: http:// www.sld.cu/galerias/pdf/sitios/gericuba/fenotipo_frailty.pdf.

13. Fabrício-Wehbe SCC, Shciaveto FV, Vendrusculo TRP, Haas VJ, Dantas RAS,
Rodrigues RAP. Adaptação cultural e validade da Edmonton Frail Scale - EFS em uma amostra de idosos brasileiros. Rev Latino-am Enfermagem. [Internet]. 2009 [2018 Jul 10]; 17(6):1043-9. Disponível em: http://www.scielo.br/scielo. php?script=sci_arttext\&pid=S0104-11692009000600018\&lng=en. doi: http:// dx.doi.org/10.1590/S0104-11692009000600018.

14. Santiago LM, Luz LL, Mattos IE, Gobbens RJJ. Adaptação transcultural do instrumento Tilburg Frailty Indicator (TFI) para a população brasileira. Cad Saúde Pública. [internet]. 2012 [acesso 2018 Jul 11]; 28(9): 1795-1801. Disponível em: http://www.scielo.br/scielo.php?script=sci_arttext\&pid=S0102311X2012000900018\&lng=en doi: http://dx.doi.org/10.1590/S0102$311 \times 2012000900018$.

15. Sewo Sampaio PY, Sampaio RA, Yamada M, Ogita M, Arai H. Validation and translation of the Kihon Checklist (frailty index) into Brazilian Portuguese. Geriatr Gerontol Int. [Internet]. 2014 [acesso 2018 Jul 11]; 14(3): 561-9. Disponível em: https://www.ncbi.nlm.nih.gov/pubmed/23992357. doi: http:// dx.doi.org/10.1111/ggi.12134. 